\title{
Towards Optimal Power Splitting in Simultaneous Power and Information Transmission
}

\author{
Yang $\mathrm{Mi}^{\dagger}$, Chunbo Luo ${ }^{\dagger *}$, Geyong Min ${ }^{\dagger}$, Pablo Casaseca-de-la-Higuera ${ }^{\ddagger}$, Zhi Wang ${ }^{+}$ \\ $\dagger$ College of Engineering, Mathematics and Physical Sciences, University of Exeter, Exeter, EX4 4QF, UK \\ $\ddagger$ Laboratory of Image Processing. ETSI Telecomunicacin, Universidad de Valladolid, 47011, Valladolid, Spain \\ + State Key Laboratory of Industrial Control Technology, Zhejiang University, China \\ E-mail: $\{y . m i$, c.luo, g.min\}@exeter.ac.uk, casaseca@lpi.tel.uva.es, zhiwangzju@qq.com
}

\begin{abstract}
Simultaneous wireless information and power transfer (SWIPT) offers novel designs that could enhance the sustainability and resilience of communication systems. Due to the very limited receiving power from radio frequency (RF) signals, optimal splitting strategies play an essential role for many SWIPT systems. This paper investigates optimal power splitting from the outage perspective by formulating the power, information and joint outage performance using a Markov chain, and studying the boundary conditions for achieving an energy-neutral state. Our results show the intrinsic trade-off between power and information outage and propose a novel polynomial method to obtain optimal power splitting. A number of experiments confirm the performance of this method.
\end{abstract}

Index Terms-Wireless power transfer, Simultaneous wireless information and power transfer, outage

\section{INTRODUCTION}

Communication systems are fundamentally controlled by energy supply and consumption [1], [2]. By studying the energy carried by radio frequency (RF) signals, wireless power transfer technologies [3], [4] have been proposed, which could potentially free communication nodes from their energy dependence on battery or the deployed environment, and facilitate broader deployment of wireless communication systems, particularly in extreme applications or disruptive environments that lack sustainable power supply or which is not easy to install or exchange batteries.

Existing studies on energy harvesting from RF signals have laid a solid foundation for SWIPT [5], [6]. The key to SWIPT is to optimally exploit the power for both energy harvesting and information processing. Power splitting (PS) proposed in [5] could achieve the energy-capacity trade-off and has been extended to wide applications such as cognitive networks [7], relaying networks [8], and frequencydivision multiplexing (OFDM) systems [9] in view of its great potential. Integrating information and power metrics as a whole to optimise the design of SWIPT systems is gaining increasing attention, which is particularly important considering the limited power carried by RF signals [8], [10].

The performance of RF powered systems has been extensively studied in literature, e.g. [11]-[13]. Authors of [12] considered the scenario where SWIPT has the opportunity to harvest energy from ambient signals, and the boundary conditions for achieving an energy neutral state within a wireless sensor network are investigated in [11]. In order to evaluate the performance of a system with the SWIPT capacity, [5] proposed to use energy-capacity trade-offs and [14] evaluated the outage probabilities. Furthermore, the studies from [6], [12] show that power splitting (PS) based structure presents high potential in building practical SWIPT systems. Several power splitting strategies have been studied. For example, authors of [15] introduced an arbitrary ratio policy, which has inferior performance due to the circuit power consumption that affects the system's overall power needs. An improved PS scheme was proposed in [16], which maximises the information throughput by adjusting the PS ratio to optimise the ergodic capacity. Information outage and power outage are usually considered separately in existing literature, leaving a significant performance gap.

Motivated by existing wireless power transfer study [17], [18], this paper focuses on the following questions: 1) What is the outage performance if both information and power outage is jointly considered? 2) How to achieve optimal power splitting from the outage perspective? The answers to these questions could provide significant contribution for both theoretical study and practical design, since the energy carried by RF signals is usually small, and SWIPT systems are usually targeting low-power applications. To achieve the goals, this paper analyses the joint outage probability under different settings in terms of battery/super capacitor size, data rate and power splitting factor, and presents a low complexity approach to achieve optimal power splitting under different transmitting power, propagation distance, and harvesting efficiency, which incurs only polynomial computation.

The rest of the paper is as follows: Section II introduces the energy harvesting model and consumption model. Section III investigates both the expected power harvesting and consumption case and outage probability. Section IV analyzes the performance of such a system under different system settings and verifies the theoretical work in Section III. Finally, Section V concludes this paper. 


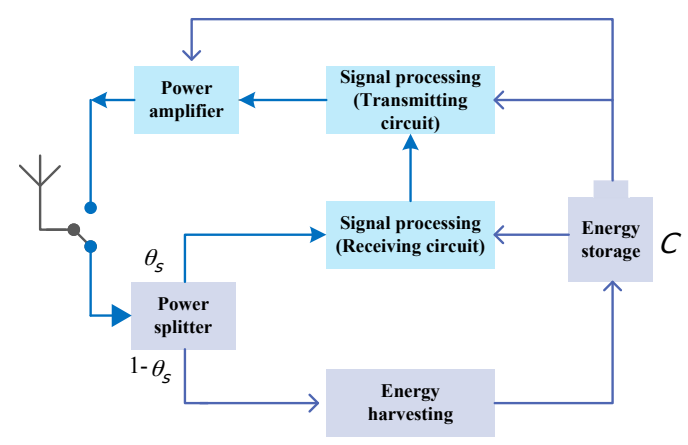

Fig. 1. The SWIPT system model.

TABLE I. MAIN SYSTEM PARAMETERS.

\begin{tabular}{l|l}
\hline $\begin{array}{l}\text { Para- } \\
\text { meters }\end{array}$ & Description and exemplar values \\
\hline$P$ & Source transmitting power $(1 \mathrm{~W})$ \\
$\theta_{s}$ & Power splitting factor $(0.5)$ \\
$\lambda$ & Expected channel gain $(1)$ \\
$d$ & Transceiver distance $(3.8 \mathrm{~m})$ \\
$\alpha$ & Path loss factor $(2)$ \\
$\beta$ & Battery conversion efficiency $(0.9)$ \\
$\eta$ & Harvesting efficiency $(1)$ \\
$T_{s}$ & Symbol duration \\
$R$ & (for a BPSK symbol: $1 /(256 \mathrm{Kbps}))$ \\
$E_{e l e c}$ & Data rate per Hz $(1 \mathrm{bit} / \mathrm{Hz} / \mathrm{s})$ \\
$E_{a m p}$ & Transmitting/receiving electronics energy \\
\hline
\end{tabular}

\section{SYSTEM MODEL}

\section{A. Energy harvesting model}

The general system model is illustrated in Fig. 1, which shows a wireless node with both transmitting and receiving capabilities, a power splitter with splitting factor $\theta_{s}$, an energy harvesting module with initial power $C$ and a storage module. $\theta_{s}$ adjusts the energy flow for both information processing and energy harvesting according to [6]. The harvested energy will be stored in the energy storing unit to feed power to the signal processing unit. Our system is modelled as follows and the implementation can be found in [6].

$$
y_{s}=\sqrt{\theta_{s}}\left(\frac{\sqrt{P} h x_{s}}{\sqrt{1+d^{\alpha}}}+n_{a}\right)+n_{p},
$$

where $P$ denotes the transmitting power from the source, e.g. a base station or a wireless router, $h$ denotes the channel coefficient from a transmitter to receiver, $x_{s}$ is the source signal with unit power, $d$ is the distance between the transmitter and receiver, and $\alpha$ is the path loss factor. The system experiences two main types of noise: the antenna unit noise $n_{a}$ and the signal processing unit noise $n_{p}$, modelled by Gaussian Distributions $N\left(0, \sigma_{a}^{2}\right)$ and $N\left(0, \sigma_{p}^{2}\right)$, respectively.
The channel fading model is assumed to follow Rayleigh distribution in the follow-on analysis for its suitability to model a typical rich scattering environment [19].

The harvested energy can be expressed as follows

$$
E_{s}=\eta\left(1-\theta_{s}\right)\left(\frac{P|h|^{2}}{1+d^{\alpha}}+\sigma_{a}^{2}\right) T_{s},
$$

where $\eta$ denotes the harvesting efficiency, and $T_{s}$ is the symbol duration. Since $\sigma_{a}^{2}$ is much smaller than the signal processing unit noise, it is neglected according to [5].

The stored energy at the battery or super capacitor is given below, subject to a conversion loss,

$$
E_{b}=\beta E_{s}=\beta \eta\left(1-\theta_{s}\right) P T_{s}\left(\frac{|h|^{2}}{1+d^{\alpha}}\right),
$$

where $\beta$ is the conversion efficiency factor.

\section{B. Energy consumption model}

We mainly consider the energy consumption of wireless sensors, consisting of three parts: the transmitter and receiver electronic circuits' consumption and the power disseminated into space. The energy consumed in reception for one bit is denoted as $E_{\text {elec }}$. The one consumed when transmitting one bit is denoted as $E_{\text {elec }}+d^{2} E_{a m p}$, where $E_{a m p}$ denotes the energy dissipating factor for ensuring an acceptable signal to noise level. $E_{\text {comp }}$ is the energy consumed by the computing subsystem.

The total energy required by a node to exchange one message with $N$ bits - a node receives one message, and then transmits its response back - can be expressed as follows:

$$
E_{c}=2 N E_{\text {elec }}+N d^{2} E_{a m p}+E_{\text {comp }},
$$

where the energy consumed by transmitter and receiver is $E_{\text {elec }} * N$ respectively.

The above equation generalises the typical energy consumption that a wireless device can incur. This can however be altered to suit more specific wireless system settings. For example, a wireless sensor node may only invoke a transmitter or receiver for certain type of operations, e.g. broadcasting.

\section{HARVESTING YIELD, CONSUMPTION AND OUTAGE}

\section{A. Expected yield and consumption}

We formulate the problem whether the power consumption of a sensor node can be sustainably supported by the harvested RF energy as follows,

$$
\begin{cases}\mathrm{E}\left\{E_{b}\right\} \geq \mathrm{E}\left\{E_{c}\right\} & \text { Sufficient } \\ \mathrm{E}\left\{E_{b}\right\}<\mathrm{E}\left\{E_{c}\right\} & \text { Insufficient }\end{cases}
$$

where $\mathrm{E}\left\{E_{b}\right\}$ denotes the expected yield, and $\mathrm{E}\left\{E_{c}\right\}$ denotes the expected consumption.

We can deduce from (3) and (4) that power harvesting is more sensitive to distance than consumption. In a practical system, power may fluctuate due to channel fading and quite often, the available energy may be insufficient to 


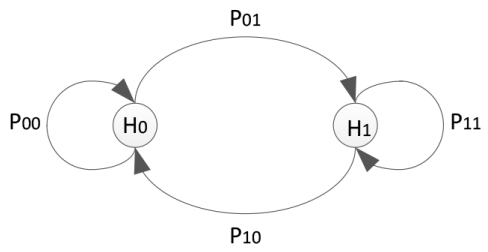

Fig. 2. The Markov Chain model of a wireless node without battery ( $H_{0}$ : the power outage state; $H_{1}$ : the power neutral state).

support communications. It is thus of more importance to evaluate the outage probabilities.

\section{B. Power outage performance}

We denote the energy states of a wireless node using a Markov Chain as in Fig. 2. Each node may experience one of the following two states at each time slot: sufficient power $\left(H_{1}\right)$ and insufficient power $\left(H_{0}\right)$. The transition probabilities between two states $(i$ and $j$ ) are denoted as $P_{i j}$ where $i$ and $j$ are either 0 (for denoting $H_{0}$ ) or 1 (for denoting $H_{1}$ ). The total number of continuous messages (receiving and transmission afterwards) is $K$. Two types of evolution of the probability values are analysed as follows:

1) No energy storage unit: If $C=0$, the above Markov Chain would reach a steady state given as follows

$$
\left\{\begin{array}{l}
\pi_{0} P_{00}+\pi_{1} P_{10}=\pi_{0} \\
\pi_{0} P_{01}+\pi_{1} P_{11}=\pi_{1}
\end{array}\right.
$$

where $\pi_{0}$ and $\pi_{1}$ are the steady-state probabilities of the two states $H_{0}$ and $H_{1}$. It can be easily inferred from the model definition that $\pi_{0}+\pi_{1}=1, P_{00}+P_{01}=1$ and $P_{10}+P_{11}=1$. Therefore we can get the overall power outage probability as follows:

$$
P_{p, o}=1-\pi_{1}=1-\frac{P_{01}}{P_{01}+P_{10}}
$$

The two transition probabilities within the above equation are expressed using instantaneous power harvesting yield and consumption:

$$
\left\{\begin{array}{l}
P_{01}=\operatorname{Pr}\left\{E_{b} \geq E_{c}\right\} \\
P_{10}=\operatorname{Pr}\left\{E_{b}<E_{c}\right\}
\end{array}\right.
$$

We introduce $g=|h|^{2}$ to denote the channel gain, which follows the exponential distribution with a probability density function (PDF) of $f_{\lambda}(g)=\lambda e^{-\lambda g}$ [1]. Therefore,

$$
\begin{aligned}
P_{01} & =\operatorname{Pr}\left\{\beta \eta\left(1-\theta_{s}\right) P T_{s}\left(\frac{|h|^{2}}{1+d^{\alpha}}\right) \geq E_{c}\right\} \\
& =\int_{E_{c} / \gamma}^{+\infty} \lambda e^{-\lambda g} \mathrm{~d} g=\exp \left\{-\frac{\lambda E_{c}}{\gamma}\right\},
\end{aligned}
$$

where $\gamma=\frac{\beta \eta\left(1-\theta_{s}\right) P T_{s}}{1+d^{\alpha}} . P_{10}$ is thus obtained as

$$
P_{10}=1-\exp \left\{-\frac{\lambda E_{c}}{\gamma}\right\} \text {. }
$$

We can obtain the steady-state power neutral probability

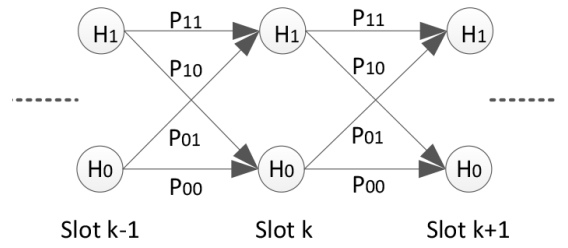

Fig. 3. The Markov Chain model of a wireless node with a rechargeable battery $\left(H_{0}\right.$ : Outage, and $H_{1}$ : Neutral).

as $\pi_{1}=\exp \left(-\frac{\lambda E_{c}}{\gamma}\right)$, and the overall outage probability for functioning $K$ time slots continuously as

$$
P_{p, o}=1-\prod_{j=1}^{K} \pi_{1}=1-\exp \left\{-\frac{\lambda K E_{c}}{\gamma}\right\} .
$$

2) With an energy storage unit and initial power $C$ : A wireless node model equipped with a battery or a super capacitor with initial power $C$ is shown in Fig. 3, where the states of a wireless node at the $(k-1)$ th, $k$ th and $(k+1)$ th time slot are shown.

In order to calculate the outage, neutral state $P_{11}(k)$ is worked out first as follows

$$
\begin{aligned}
P_{11}(k) & =\operatorname{Pr}\left\{\sum_{j=1}^{k} \beta \eta\left(1-\theta_{s}\right) P T_{s}\left(\frac{g_{j}}{1+d^{\alpha}}\right)+C \geq k E_{c}\right\} \\
& =\operatorname{Pr}\left\{\sum_{j=1}^{k} g_{j} \geq \frac{k E_{c}-C}{\gamma}\right\} .
\end{aligned}
$$

If $C \geq k E_{c}$, the above probability would remain $100 \%$.

Let $G(k, \lambda)=\sum_{j=1}^{k} g_{j}=x$. Since $g_{j}$ follows an exponential distribution, $G(k, \lambda)$ follows an Erlang distribution [20] with its PDF as

$$
f(x)=\frac{\lambda^{k} x^{k-1} e^{-\lambda x}}{(k-1) !}
$$

We can then obtain the following closed form

$$
\begin{aligned}
P_{11}(k) & =\int_{\frac{k E_{c}-C}{\gamma}}^{\infty} f(x) \mathrm{d} x \\
& =\sum_{n=0}^{k-1} \frac{1}{n !} e^{-\frac{\lambda\left(k E_{c}-C\right)}{\gamma}}\left(\frac{\lambda\left(k E_{c}-C\right)}{\gamma}\right)^{n}
\end{aligned}
$$

The overall outage for the node to function $K$ time slots continuously can be expressed as follows

$$
P_{p, o}=1-\prod_{k=1}^{K} P_{11}(k) .
$$

How much initial power is required for achieving the minimum outage probability?

Proposition 3.1: The condition to achieve minimum power outage probability is that the initial power of wireless node meets the total power consumption of $K$ continuous 
functioning time slots: $C=K E_{c}$, and the minimum power outage probability is 0 .

Proof: The result is obtained by taking the derivative of $P_{11}(k)$. By allocating $\frac{\partial P_{p, o}(k)}{\partial C}=0$, we have $C=k E_{c}$.

However, practical systems usually allow outage occurrence at a certain probability level, which can be calculated by (14). Another important information is the residual power left in the energy storage unit after $K$ time slots, which provides the foundation of the next $K$ time slots. The following proposition gives the answer.

Proposition 3.2: The average power after $K$ continuous time slots is $C_{r e s}=\gamma K / \lambda-K E_{c}+C$. as follows

Proof: The proof of Proposition 3.2 is straightforward

$$
C_{r e s}=\mathrm{E}\left\{E_{b}-K E_{c}+C\right\}=\gamma K / \lambda-K E_{c}+C .
$$

From Proposition 3.2, a sustainable wireless node can be built only if $\gamma / \lambda \geq E_{c}$. Otherwise, the whole system would gradually deteriorate to the case $C=0$ in Section III-B1, thus generating significantly higher outage probability.

\section{Information outage performance}

The ultimate purpose of harvesting power is to exchange information. It is thus important to evaluate the probability of information outage under the condition that only partial receiving power can be used for information detection, which can be formulated by the probability that the capacity of a giving channel is below the required data rate $R$. A similar Markov model to Fig. 1 can be introduced to express the transition of different states.

We firstly denote the equivalent SNR at the receiver side as (The source SNR is denoted as $\rho=P / \sigma_{p}^{2}$ ),

$$
\mathrm{SNR}=\frac{\theta_{s} P|h|^{2}}{\left(1+d^{\alpha}\right) \sigma_{p}^{2}}
$$

The outage probability is thus formulated as follows

$$
P_{10}^{\prime}=\operatorname{Pr}\{\log (1+\mathrm{SNR})>R\},
$$

and the steady-state probability

$$
\pi_{1}^{\prime}=\frac{P_{01}^{\prime}}{P_{01}^{\prime}+P_{10}^{\prime}}
$$

where the probability is taken over all possible $g=|h|^{2}$, given below

$$
P_{10}^{\prime}=\int_{0}^{\frac{\left(1+d^{\alpha}\right)\left(2^{R}-1\right)}{\theta_{s} \rho}} f_{\lambda}(g) \mathrm{d} g
$$

and $P_{01}^{\prime}+P_{00}^{\prime}=1$ and $P_{10}^{\prime}+P_{11}^{\prime}=1$.

The closed form of the information outage is as follows,

$$
P_{i, o}=1-\prod_{j=1}^{K} \pi_{1}^{\prime}=1-\exp \left\{-\lambda K\left(1+d^{\alpha}\right) \frac{\left(2^{R}-1\right)}{\theta_{s} \rho}\right\}
$$

\section{Joint outage performance}

The joint power and information outage of this system can provide a full picture of the overall performance of the system. At any time slot $k$, the joint outage can be expressed as follows,

$$
P_{o}(k)=1-\left(1-P_{p, o}(k)\right)\left(1-P_{i, o}(k)\right)
$$

where $P_{p, o}(k)$ is the power outage at time $k$, given by

$$
\begin{aligned}
P_{p, o}(k) & =1-P_{11}(k) \\
& =1-\sum_{n=0}^{k-1} \frac{1}{n !} e^{-\frac{\lambda\left(k E_{c}-C\right)}{\gamma}}\left(\frac{\lambda\left(k E_{c}-C\right)}{\gamma}\right)^{n}
\end{aligned}
$$

and $P_{i, o}(k)$ is the information outage at time $k$, given by

$$
P_{i, o}(k)=1-\exp \left\{-\lambda\left(1+d^{\alpha}\right) \frac{\left(2^{R}-1\right)}{\theta_{s} \rho}\right\}
$$

There exists a trade-off between power outage and information outage, which is primarily controlled by the power splitting factor $\theta_{s}$.

Theorem 3.3: The ideal power splitting factor $\theta_{s}$ for a minimum overall outage probability, represented as $\theta_{s}=$ $1-x$, is given by the roots of the following polynomial,

$$
\sum_{n=0}^{k-1} \frac{\epsilon \xi^{n-k}(k-1) !}{n !} x^{k+1-n}+x^{2}-2 x+1=0 .
$$

where $\epsilon=\frac{-\lambda\left(1+d^{\alpha}\right)\left(2^{R}-1\right)}{\rho}$, and $\xi=\frac{\lambda\left(k E_{c}-C\right)\left(1+d^{\alpha}\right)}{\beta \eta P T}$.

Proof: We firstly obtain the derivative of $P_{o}(k)$ against $\theta_{s}$. By letting $\frac{\partial P_{o}(k)}{\partial \theta_{s}}=0$, we can work out an expression of $x$ and $\theta_{s}$ which can be easily solved out by calculating the roots of a single-variable polynomial.

\section{NUMERICAL EXPERIMENTS}

This section presents numerical experiments to investigate the power and information outage performance of a wireless node supported by SWIPT. The experiments aim to provide insight on how to optimise a sustainable RF energy harvesting node. Most of the parameters are listed in Table I, with a few exceptions that are specifically described in the relevant experiments.

\section{A. Power outage investigation}

The first experiment examines the outage of the continuous working case, by fixing the distance to $10 \mathrm{~m}$, which is slightly greater than the neutral case and adjusting $\theta_{s}$. Fig. 4 depicts the results. The power outage increases with the continuous working time $K$, where lower $\theta_{s}$ can contribute to a better outage performance. However, the information outage would increase accordingly. A better solution is to equip a rechargeable battery or super capacitor with some initial power.

Fig. 5 demonstrates the case with initial power and a rechargeable battery or super capacitor. We fix the distance to $4 m$ and adjust the initial power $C$, from which we can 


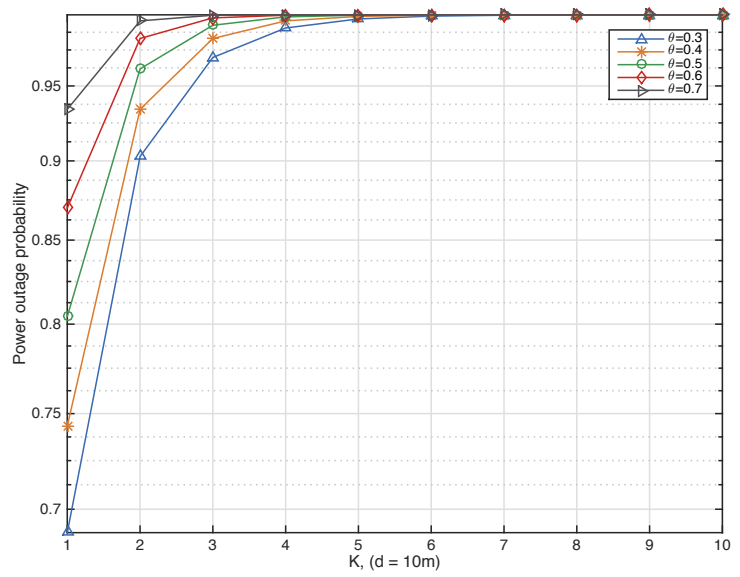

Fig. 4. Power outage probability against the continuous functioning time slots $K$ in the case of no battery.

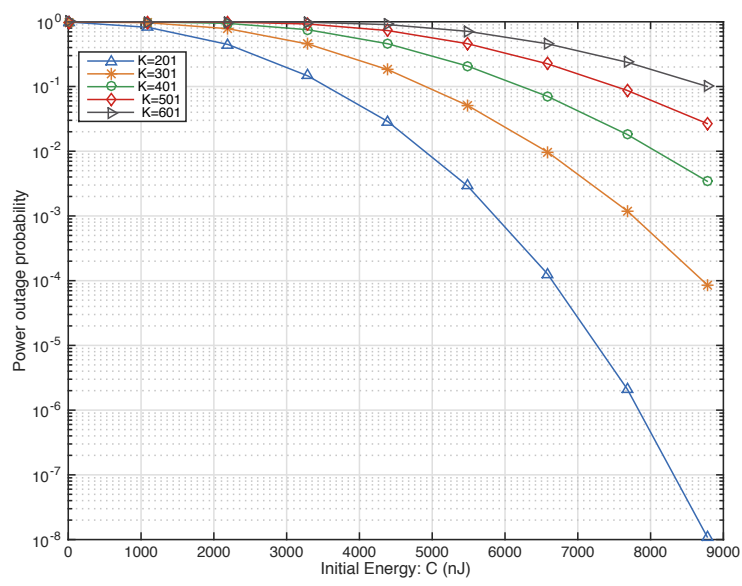

Fig. 5. Continuous power outage probability against initial power.

see an obvious performance improvement: even for $K=$ 601 , the power outage probability can reach $10^{-1}$.

To wrap up this subsection, the final experiment studies the change of distance and power splitting factor towards the power outage performance. We fix the initial battery power to $1500 n J$ (Case 2), and choose $K=32$. Fig. 6 presents the results. Two observations can be obtained: 1) the power splitting factor $\theta_{s}$ has significant impact in outage performance: at the same distance, e.g. $d=15 \mathrm{~m}$, the outage probability for $\theta_{s}=0.7$ (Case 2) is almost $100 \%$, while that for $\theta_{s}=0.3$ (Case 1 ) is approximately $10^{-1} ; 2$ ) lower distance and higher $\theta_{s}$ are the key to optimal power outage performance.

\section{B. Information outage investigation}

The next group of experiments focus on information outage to measure the probability of exchanging information successfully.

Fig. 7 reveals the information outage vs SNR for different $\theta_{s}$ values. As expected, the increase in SNR leads to lower information outage. The increase of power share from the received signal also contributes to lower outage. The

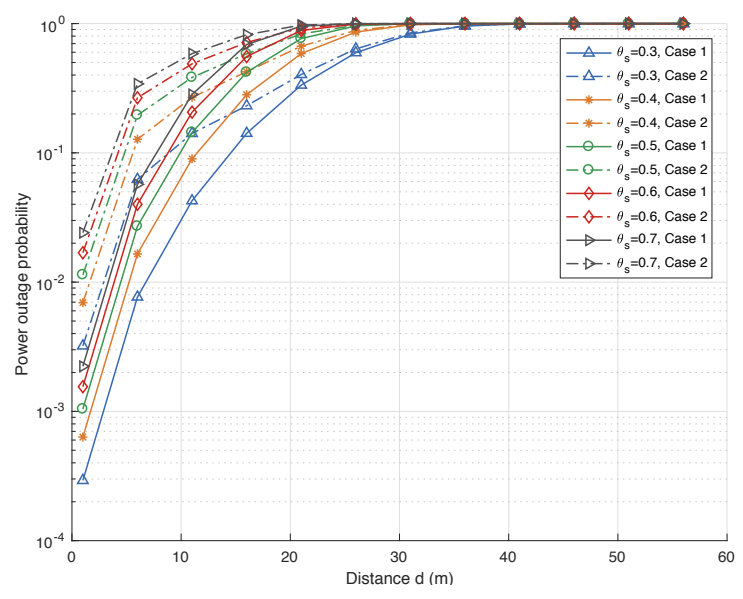

Fig. 6. Power outage probability under varied distance and power splitting factor (Case 1: without $C$, and Case 2: with initial $C$ ).

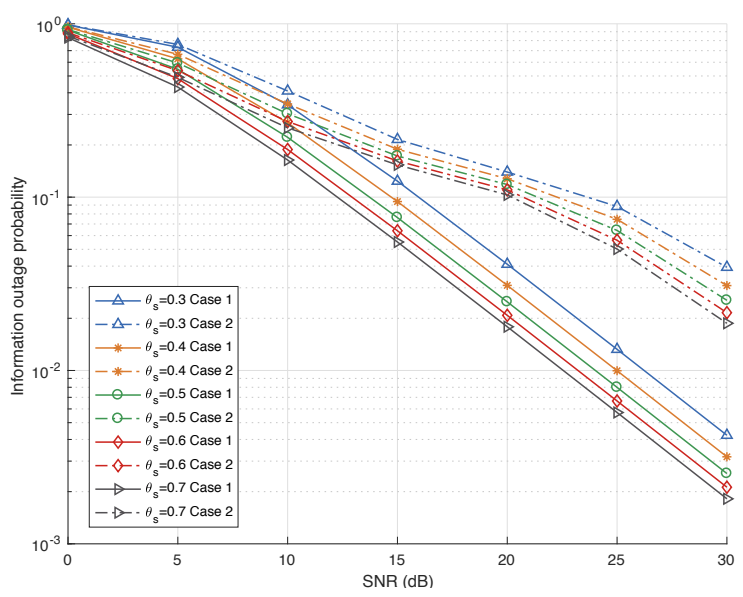

Fig. 7. Information outage probability against SNR.

increment becomes less significant when $\theta_{s}$ is greater, e.g. $0.5 \sim 0.9$.

Compared to the power outage in Fig. 6, information outage has a smoother impact on distance changes. Fig. 8 shows some examples when SNR is set to $30 d B$. Generally, smaller distance contributes to lower outage probability, and the increasing tread is similar for different $\theta_{s}$ values.

\section{Joint outage and theoretical $\theta_{s}$}

The previous two groups of experiments have considered both edges of the sword, and this subsection aims to see the combined effects by evaluating the joint outage performance against the power splitting factor. The experiment also verifies whether (23) holds or not.

Fig. 9 gives an overall view under neutral state, where both $K$ and $C$ are changed. The curves represent the joint outage probability, which resemble a bowl shape where its lowest point indicates the place of the ideal $\theta_{s}$. From the results, we can see Theorem 3.3 has been confirmed. All cases further show that higher $C$ decreases the overall outage probability significantly, and also reveal that more 


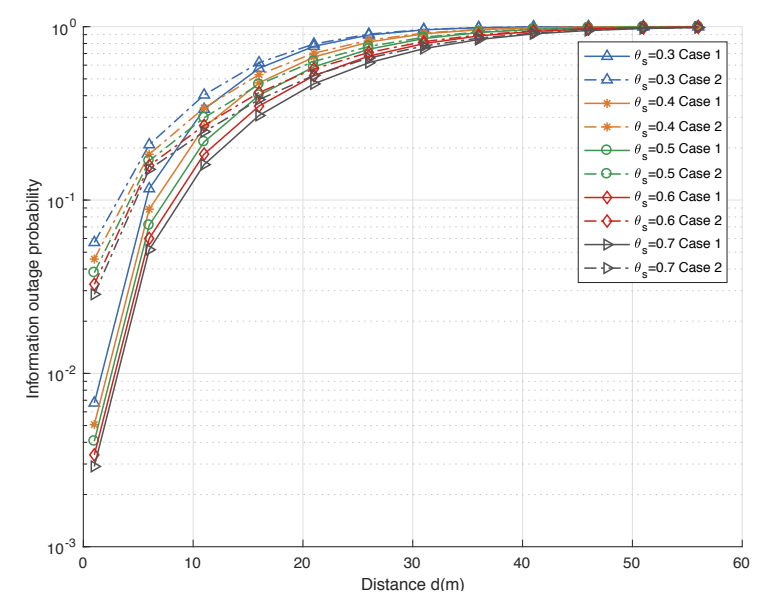

Fig. 8. Information outage probability against distance.

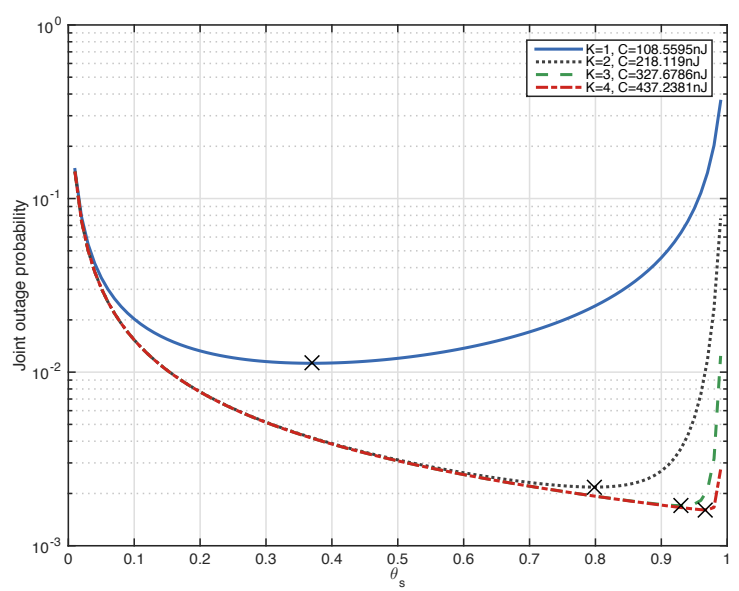

Fig. 9. Joint power and information outage. The symbol $\times$ denotes the theoretical $\theta_{s}$ values that gives the minimum outage, calculated by (23).

power should be used for information processing if there is some existing energy in the storage unit.

\section{CONCLUSION}

This paper investigates the optimal power splitting under joint power and information outage consideration. Results can be summarised by the following points: 1) The battery power has significant impact on the overall power outage of such a wireless transfer system; 2) The theoretical instantaneous outage could reach the minimum if there is sufficient initial power; 3) The energy sustainable state can be maintained if there is sufficient residual power $C_{r e s}$; 4) The ideal power splitting factor $\theta_{s}$ can be calculated through a polynomial equation. This method can be applied in the SWIPT system to improve the overall outage performance.

\section{ACKNOWLEDGMENT}

This paper is sponsored by the Royal Society-MOST Grant (No. 185730) and the Royal Society of EdinburghNSFC Grant (NNS/INT 15-16 Casaseca). All code is available under request.

\section{REFERENCES}

[1] J. G. Proakis, Digital communications, 4th ed. New York: McGrawHill, Inc., 2001.

[2] H. Jayakumar, K. Lee, W. S. Lee, A. Raha, Y. Kim, and V. Raghunathan, "Powering the internet of things," in 2014 IEEE/ACM International Symposium on Low Power Electronics and Design (ISLPED), Aug. 2014, pp. 375-380.

[3] P. Grover and A. Sahai, "Shannon meets tesla: Wireless information and power transfer," in 2010 IEEE International Symposium on Information Theory, Jun. 2010, pp. 2363-2367.

[4] L. R. Varshney, "Transporting information and energy simultaneously," in 2008 IEEE International Symposium on Information Theory, Jul. 2008, pp. 1612-1616.

[5] X. Zhou, R. Zhang, and C. K. Ho, "Wireless information and power transfer: Architecture design and rate-energy tradeoff," in 2012 IEEE Global Communications Conference (GLOBECOM), Dec. 2012, pp. 3982-3987.

[6] R. Zhang, R. G. Maunder, and L. Hanzo, "Wireless information and power transfer: from scientific hypothesis to engineering practice," IEEE Commun. Mag., vol. 53, no. 8, pp. 99-105, Aug. 2015.

[7] M. Amini, M. Mahdavi, and M. J. Omidi, "Energy efficiency optimization of secondary network considering primary user return with alternating-phase-type traffic," IEEE Trans. Commun., vol. PP, no. 99 , pp. 1-1, 2017.

[8] D. Mishra and S. De, "i2res: Integrated information relay and energy supply assisted rf harvesting communication," IEEE Trans. Commun., vol. 65, no. 3, pp. 1274-1288, March 2017.

[9] K. Huang and E. Larsson, "Simultaneous information and power transfer for broadband wireless systems," IEEE Trans. Signal Process., vol. 61, no. 23, pp. 5972-5986, Dec. 2013.

[10] Y. Liu and X. Wang, "Information and energy cooperation in OFDM relaying: Protocols and optimization," IEEE Trans. Veh. Technol., vol. PP, no. 99, pp. 1-1, 2015 .

[11] S. Peng, T. Wang, and C. Low, "Energy neutral clustering for energy harvesting wireless sensors networks," $\mathrm{Ad}$ Hoc Networks, vol. 28, pp. 1 - 16, 2015. [Online]. Available: http: //www.sciencedirect.com/science/article/pii/S1570870515000062

[12] X. Lu, I. Flint, D. Niyato, N. Privault, and P. Wang, "Self-sustainable communications with RF energy harvesting: Ginibre point process modeling and analysis," IEEE J. Sel. Areas Commun., vol. 34, no. 5, pp. 1518-1535, May 2016.

[13] S. Ulukus, A. Yener, E. Erkip, O. Simeone, M. Zorzi, P. Grover, and K. Huang, "Energy harvesting wireless communications: A review of recent advances," IEEE J. Sel. Areas Commun., vol. 33, no. 3, pp. 360-381, Mar. 2015.

[14] Z. Ding, I. Krikidis, B. Sharif, and H. V. Poor, "Wireless information and power transfer in cooperative networks with spatially random relays," IEEE Trans. Wireless Commun., vol. 13, no. 8, pp. 44404453, Aug. 2014.

[15] R. Zhang and C. K. Ho, "Mimo broadcasting for simultaneous wireless information and power transfer," IEEE Trans. Wireless Commun., vol. 12, no. 5, pp. 1989-2001, May 2013.

[16] X. Zhou, "Training-based SWIPT: Optimal power splitting at the receiver," IEEE Trans. Veh. Technol., vol. 64, no. 9, pp. 4377-4382, Sept. 2015.

[17] V. Liu, A. Parks, V. Talla, S. Gollakota, D. Wetherall, and J. R. Smith, "Ambient backscatter: Wireless communication out of thin air," SIGCOMM Comput. Commun. Rev., vol. 43, no. 4, pp. 39-50, Aug. 2013. [Online]. Available: http://doi.acm.org/10.1145/ 2534169.2486015

[18] B. Allen, T. Ajmal, V. Dyo, and D. Jazani, "Harvesting energy from ambient radio signals: A load of hot air?" in Proceedings of Antennas and Propagation Conference (LAPC), 2012 Loughborough, Nov. 2012, pp. 1-4.

[19] G. Pan, H. Lei, Y. Yuan, and Z. Ding, "Performance analysis and optimization for swipt wireless sensor networks," IEEE Trans. on Commun., vol. 65, no. 5, pp. 2291-2302, May 2017.

[20] M. Evans, N. Hastings, and B. Peacock, Erlang Distribution., ser. Chapter 12, Statistical Distributions. New York: Wiley, 2000. 